\title{
Extra-Pulmonary Tuberculosis: Retrospective Review of 83 Confirmed Cases, Observed in Radiology in Lomé (TOGO)
}

\author{
Mazamaesso Tchaou ${ }^{{ }^{*}}$, Tchin Darre ${ }^{2}$, Komi Edem Mossi ${ }^{3}$, Lantam Sonhaye ${ }^{1}$, \\ Mohaman Djibril ${ }^{3}$, Awereou Kotosso ${ }^{4}$, Lama Agoda-Koussema1, \\ Komlan Adjenou' ${ }^{1}$, Koffi N'Dakena ${ }^{1}$ \\ ${ }^{1}$ Department of Radiology, Sylvanus Olympio Teaching Hospital of Lomé, Lomé, Togo \\ ${ }^{2}$ Department of Pathology Laboratory, Sylvanus Olympio Teaching Hospital of Lomé, Lomé, Togo \\ ${ }^{3}$ Department of Internal Medicine, Sylvanus Olympio Teaching Hospital of Lomé, Lomé, Togo \\ ${ }^{4}$ Department of Infectious and Tropical Diseases, Sylvanus Olympio Teaching Hospital of Lomé, Lomé, Togo \\ Email: "joseph_tchaou@yahoo.fr
}

Received 1 February 2016; accepted 23 March 2016; published 28 March 2016

Copyright (C) 2016 by authors and Scientific Research Publishing Inc.

This work is licensed under the Creative Commons Attribution International License (CC BY). http://creativecommons.org/licenses/by/4.0/

c) (i) Open Access

\section{Abstract}

Background: Tuberculosis primarily affects lungs, but all organs may be affected. Extra-pulmonary forms of tuberculosis cause a big public health problem as it is diagnosed late. This delay in diagnosis is not only due to the delay in the examination, but also due to difficulties related to extrapulmonary tuberculosis diagnostic. Aim: To describe the different locations of extra-pulmonary Tuberculosis and its aspects in Togo. Patients and Methods: A retrospective analysis of all extrapulmonary tuberculosis cases diagnosed by imaging over five years (January 2010 to December 2015) was done. All the cases were confirmed either by direct examination of biological fluids or by histological analysis of biopsy and surgical samples or by culture on specific area. Data were collected from the Department of Radiology, from Microbiology Laboratory of the Pneumo-phthisiology unit and from Pathology Laboratory of Sylvanus Olympio Teaching Hospital of Lomé. Results: A total of 83 cases of extra-pulmonary Tuberculosis had been taken into account: 45 men and 38 women, with a maximum number of cases $(66.3 \%)$ in the age group between 20 to 40 years old. An HIV infection was known in 51 patients (61.4\%). Pulmonary damage was associated in 21 patients $(25.3 \%)$. A total of 116 extra-pulmonary tuberculosis locations were recorded. Damages were pleural in $37.1 \%(43)$, ganglion in $24.1 \%(28)$, osteo-articular in $15.5 \%(18)$, abdominal in $12.9 \%(15)$, military represented $8.6 \%(10)$, a case of testicular damage, and a case of mammary tuberculosis. Conclusion: The medical scanning based on conventional radiography, ultrasonography and TDM is indispensable for the diagnosis and the care taking of extra-pulmonary tuber-

${ }^{*}$ Corresponding author.

How to cite this paper: Tchaou, M., Darre, T., Mossi, K.E., Sonhaye, L., Djibril, M., Kotosso, A., Agoda-Koussema, L., Adjenou, K. and N'Dakena, K. (2016) Extra-Pulmonary Tuberculosis: Retrospective Review of 83 Confirmed Cases, Observed in Radiology in Lomé (TOGO). Open Journal of Radiology, 6, 49-55. http://dx.doi.org/10.4236/ojrad.2016.61007 
culosis since some locations may be confused with many other diseases and lead to misdiagnosis errors and delay the treatment.

\title{
Keywords
}

\author{
Extra-Pulmonary Tuberculosis, Radiography, Ultrasonography, Computed Tomography, \\ Diagnostic, Africa, Togo
}

\section{Introduction}

Tuberculosis (TB) is a granulomatous disease caused by Mycobacterium tuberculosis. It is a world-wide public health problem, mostly in developing countries like Togo, despite the identification of the pathogenic agent over a century [1]. Tuberculosis primarily affects the lungs, but all organs may be affected [2]. Extra-pulmonary forms of tuberculosis pose a big public health problem, because unlike pulmonary forms that lead patients quickly at health centers where care is given by the national program against tuberculosis are codified and free of charge; they are commonly unrecognized or late diagnosed, which delay the treatment. This delay in diagnosis is not only due to the delay in the examination, but also due to the diagnostic difficulties related to extra-pulmonary tuberculosis. Extra-pulmonary tuberculosis accounts for $8.7 \%$ of all tuberculosis locations [3]. Medical imaging based on standard radiography, ultrasonography and computed tomography is essential to the diagnosis and sometimes to the care by interventional procedures. The aim of this study was to describe the different locations of extra-pulmonary tuberculosis and its aspects by imaging in Togo.

\section{Patients and Methods}

This is a descriptive retrospective observational study over 5 years (January 2010-December 2015) of all cases of extra-pulmonary tuberculosis diagnosed by imaging and confirmed by the direct examination of biological fluids, histology of biopsy specimens or surgical or culture on specific area. Data were collected from Archives of the Department of Radiology, Microbiology Laboratory of the Pneumo-phthisiology unit and from Pathology Laboratory of Sylvanus Olympio Teaching Hospital of Lomé. The diagnostic evidence had been provided either by searching through direct examination of BAAR in Microbiology Laboratory located in the Pneumo-phthisiology unit for the biological fluids sampling (sputum, ascites, abscesses pus, particular liquid and pleural liquid); or by culture on specific swab area whether it is liquid or biopsy specimens or finally by histological examination of surgical specimens or biopsy in search of an isolated necrosis caseous or associated with granulomas épithélioïdesgiganto-cellular to the Pathology Laboratory. The parameters studied concern the epidemiological data (age, gender), tuberculosis background, an HIV co-infection, an association with lung damage, the imaging data (means of imaging used, damage location, radiological aspects viewed, and the use or not of interventional imaging for diagnostic or therapeutic purposes.We have complied with all ethical considerations in force in Togo.

\section{Results}

During the study period, 83 patients had been diagnosed and confirmed as suffering from extra-pulmonary tuberculosis on a total of 79,616 patients seen in the department for imaging examinations (X-ray, ultrasound, CT). They were 45 men (54.2\%) and 38 women (45.8\%), accounting for 1.18 sex ratio. The average age was $32.6 \pm$ 16.3 years with 1 and 82 years old of extremes. We noticed a maximum case, 66.3\% (55 cases) in the age group between 20 - 40 years old (Figure 1).

Twenty-two patients had some tuberculosis treated past record: pulmonary $(n=15)$ and pleural $(n=5)$ ganglionic $(\mathrm{n}=2)$. Over 22 patients of pulmonary $\mathrm{x}$-ray who presented a tuberculosis past record, they were parenchymal sequel in 9 out the 15 (60\%) with a pulmonary tuberculosis past record and pleural sequel in 1 out 5 with a pleural tuberculosis past record. An HIV infection was known in 51 patients (61.4\%). Pulmonary damage was associated in 21 patients (25.3\%). Extra-pulmonary location was unique in 45 patients (54.2\%). The damages were multifocal in 38 patients (45.8\%). 


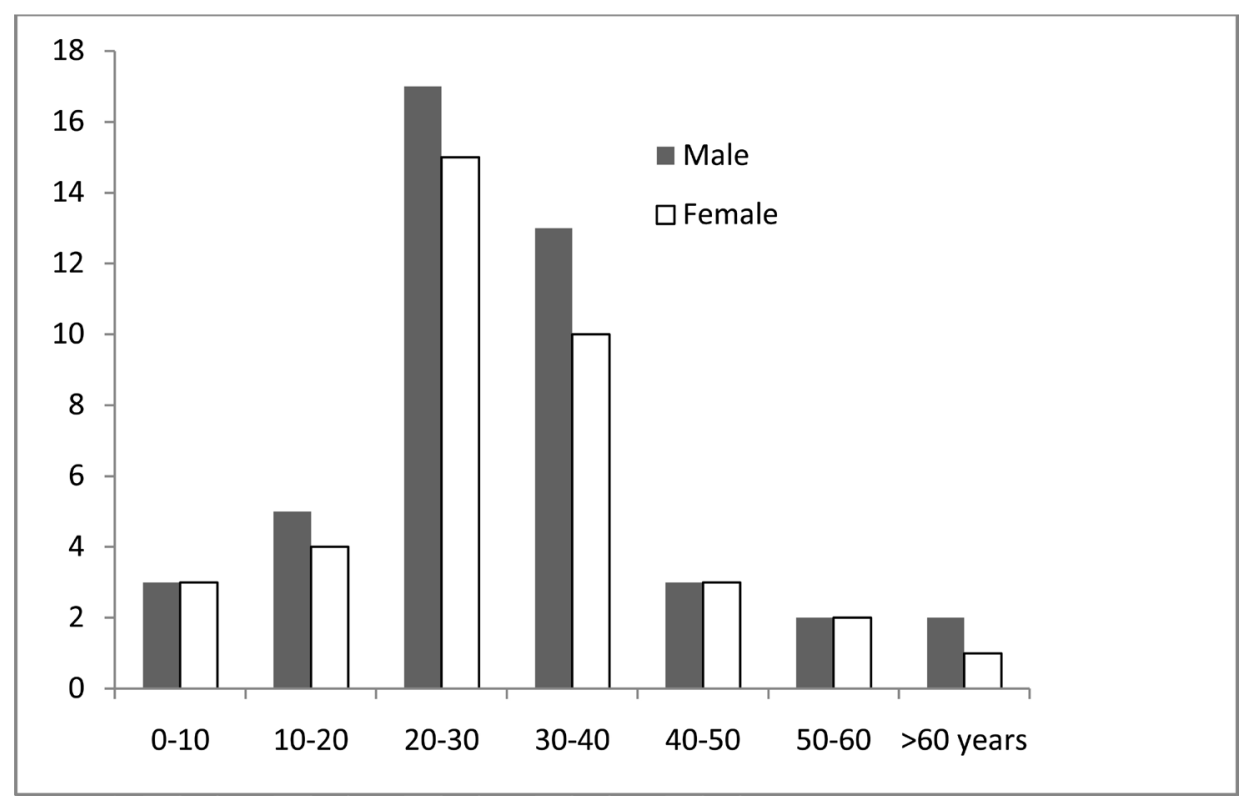

Figure 1. Distribution of extra-pulmonary tuberculosis cases by age and sex.

Table 1. Distribution of damages according to the extra-pulmonary tuberculosis location.

\begin{tabular}{cc}
\hline & Nomber (\%) \\
Pleural & $43(37.1)$ \\
Ganglion & $28(24.1)$ \\
Osteoarticular & $18(15.5)$ \\
Abdominal & $15(12.9)$ \\
Miliary & $10(08.6)$ \\
Genito-urinar & $01(0.9)$ \\
Brest & $01(0.9)$ \\
Total & $\mathbf{1 1 6 ( 1 0 0 )}$ \\
\hline
\end{tabular}

Among the 83 patients involved in the study, a total of 116 extra-pulmonary locations of tuberculosis were recorded (Table 1).

The pleural location of tuberculosis accounted for 37.1\% (43) of all locations. The thorax x- ray had enabled to diagnose pleural effusion in all patients. It was unilateral effusion for all, partitioned in 10 patients. The ultrasonography location before pleuralbiopsy was performed in 37 patients. The tuberculosis histological confirmation was made on pleural swab taken by biopsy tapping. On pulmonary x-ray, there was an association of pleural and pulmonary damage in 10 cases.

Peripheral Ganglions were affected in 15 patients. That was cervical ganglion $(n=8)$ and inguinal $(n=5)$ and axillary $(n=2)$. These peripheral Ganglionic locations were associated with other locations in 14 cases. The only case of isolated peripheral Ganglionic damage was a unique cervicaladenitis of a 7 years old child, an HIV carrier. Ultrasonography had evoked the diagnosis of adenomegaly and histology demonstrated tuberculosis damage after examination of the removed ganglion. The deep ganglion was abdomenalincoelio-mesenteric area and found by TDM and/or abdominal ultrasonography in 3 patients (Figure 2). A case of retro peritoneal adenomegaly got a guided biopsy, with histological confirmation.

Out of 18 osteo-articular tuberculosis locations in our series, the rachis $(n=12)$ represented the first location, followed by ribs $(n=2)$. Knee, hip, wrist, and sternum were damaged one in each case. The imaging was essentially based on radiography. Ultrasonography and CT-scan were useful in all cases. Indeed, in peripheral articular damages, ultrasonography had helped to highlight liquid peri-articular collections whom tapping guided by ultrasonography had enable to confirm the presence of BK. CT-scan was useful to confirm diagnosis in spondy- 
litis and had discovered psoas abscess in 8 cases which had also been taken under ultrasonography guidance for confirmation.

Abdominal tuberculosis concerned in this study the peritoneum $(n=13)$, spleen $(n=2)$ and the liver $(n=1)$. The peritoneal damage that represented $11.2 \%$ of all locations was brought up by abdominal ultrasonography in the presence of ascites. Only 5 patients had benefited from a TDM to explore their ascites. The splenic and liver damage were as micronodular lesions hypoechoic diffuse of parenchyma miliary appearance. The spleen and hepatic miliary were identified in patients with HIV.

The 10 cases of pulmonary miliary had been revealed by pulmonary X-rays before diffuse and bilateral micronodular opacities in all cases (Figure 3). The demonstration of BK in direct splits examination had confirmed the diagnosis.

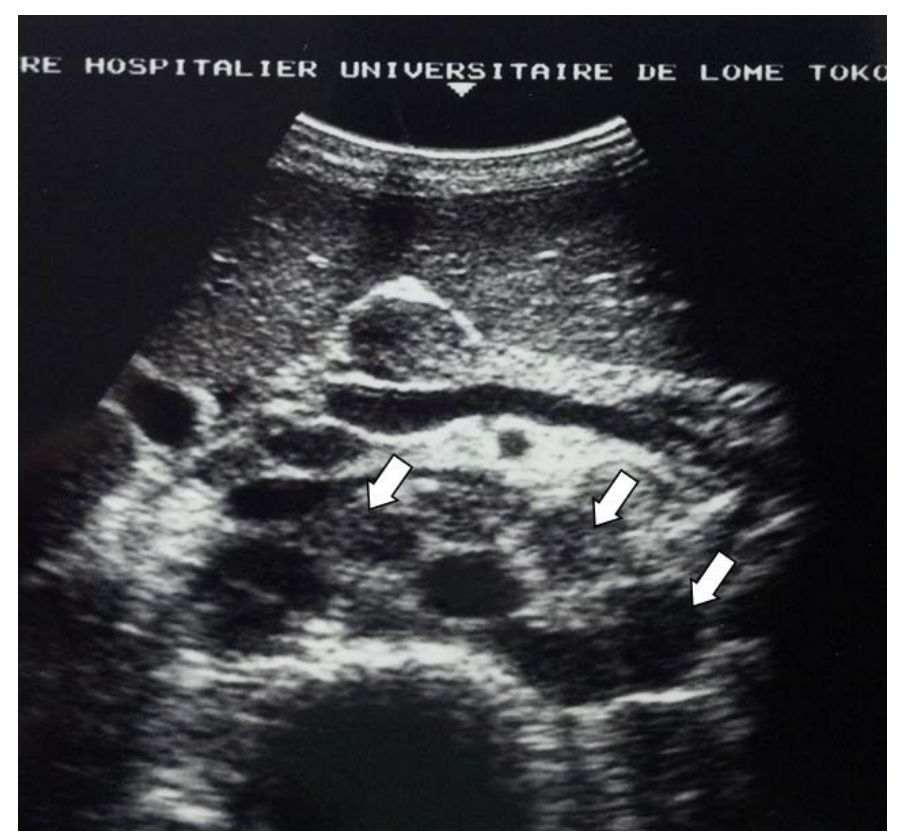

Figure 2. Ultrasonographical cut through the epigastric area showing retro-peritoneal adénomégalies (white arrows).

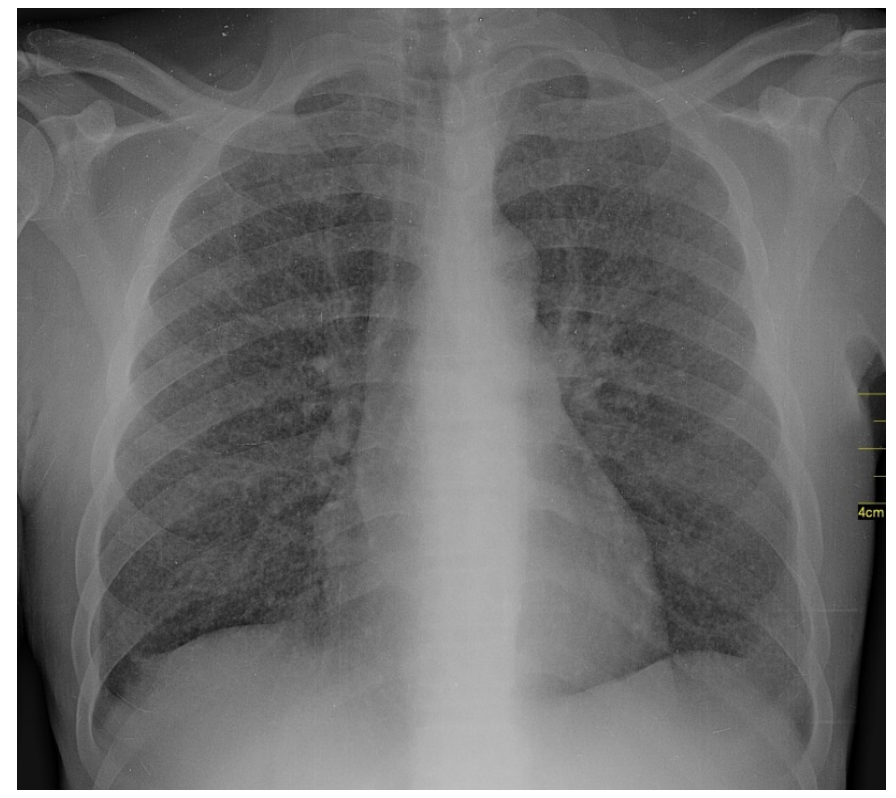

Figure 3. Front pulmonary x-rayshowing a pulmonary miliary characterized by diffuse micronodular opacities bilateral. 
The only case of genito urinary damage was atesticular location in a 27-year-old patient, HIV negative. It was a big heterogeneous left testicle in ultrasonography. Histological confirmation was made by operating room test.

A case of breast tuberculosis was identified. The diagnosis of mass breast in flammatory had been mentioned in ultrasonography. The confirmation was made by histological examination of biopsyguided of the mass achieved after failure of the first anti-inflammatory treatment and nonspecific antibiotic therapy.

\section{Discussion}

Our study is aretrospective study of extra-pulmonary tuberculosis over a period of 5 years at the Teaching Hospital of Lomé. It brought together all cases diagnosed in the department of radiology and medical imaging of Sylvanus Olympio Teaching Hospital of Lomé and confirmed either by the pathological anatomy laboratory or by the microbiology laboratory. However, we missed many cases. Those are patients who prefer go to the traditional healers, or who have completed their imaging checkup in other medical centers or those who by lack of financial means can't do the imaging tests. In addition, our study has some limitations; in fact, many cases of extra-pulmonary tuberculosis suspected by imaging could not be confirmed either by lack of samples before starting treatment or by lack of financial means to pay the cyto-pathological examinations.

In most African studies [3] [4], the majority of our patients are young, with a male predominance. Co-infection with HIV was high, up to $61.1 \%$ of cases. This rate is close to that of Darré et al. [4] who considers that HIV is changing the face of tuberculosis. Actually according to the World Health Organization (WHO), in Africa, HIV is the main cause of the increase of TB impact during the past decade [5].

Pleural tuberculosis is by far the most common extra-pulmonary location of TB [6] [7]. They accounted for $37.1 \%$ in our series, which was close to $35.6 \%$ of hospital study of Yoon et al. [7].

Ganglionic tuberculos is accounted for $24.1 \%$ in our series of extra-pulmonary locations, which placed it in second position after the pleural location. This position corresponds to its place in most of studies [8]. Ultrasonography and TDM enable to directly highlight adenomegalies directly, but also to guide biopsies of deep adenomegalies, when there was no other simple ways to confirm tuberculosis.

Oesto-articular tuberculosis accounted for $1 \%$ to $4.3 \%$ tuberculosis cases [9]-[11]. In our study, it accounts for $15.5 \%$ of all locations. This frequency is in line with the data on the topic who estimates that frequency between $10 \%$ and $15 \%$ of extra-pulmonary tuberculosis according to the authors [12] [13]. TB spinal location was the most frequent osteo-articular damage in our series. This is in line with most studies [11] [12] [14] [15].

The abdominal tuberculosis location in frequency order is the $6^{\text {th }}$ location of extra-pulmonary tuberculosis after pleural location, ganglionic, genito-urinary, osteoaricular, military and meningeal [8], it represents the fourth location in our study. This position is related to the large number of peritoneal tuberculosis cases in our series which accounted for $11.2 \%$ of all locations. Peritoneal tuberculosis is the most common form of abdominal tuberculosis [16]; it represented $7.2 \%$ of extra-pulmonary TB in a study in one of pathological rooms at the Teaching Hospital of Lomé [4]. This difference between our study and that of Darré et al. [4] could be explained by the low proportions of other locations including ganglionic, genitor-urinary and gastrointestinal diseases that were not often explored by imaging or when the radiological exploration is made, the levy for bacteriological or histological confirmation is not easy. In medical imaging, ultrasonography is the first examination to be carried out in presence of ascites. It is completed by CT-Scan in search of signs to guide the diagnosis, especially to distinguish tuberculous ascites from carcinomatous ascites and other causes including cirrhosis ascites. Although some authors believe that the distinctive elements provided by ultrasonography and CT-scan have little orientation value [17] [18], these two means remain essential in exploring ascites. Hepatic TB location is very rare [19], it was $0.9 \%$ in our series. As N'goran et al. reported [20] it was the ultrasonography that allowed evoking the diagnosis, ultrasonography-guidedbiopsy and pathological examination had enabled to confirm the origin of tuberculosis by displaying the giant cellgranuloma, with necrosis central caseous. The splenic tuberculosis location is also rare [21]. No gastrointestinal location was mentioned in this study while it represented between $3 \%$ and 5\% of extra-pulmonary tuberculosis according to Chong and Lim [22]. This lack of cases cannot be explained by the absence of suspected cases by imaging, but rather by the difficulties in swabbing the samples for histological confirmation.

A testicular location had been recorded. This TB location is rare and exceptionally isolated [23]. The ultrasonography aspect may be mistaken for atesticular tumour, especially in the young patient [24]. Like other rare or exceptional locations, in presence of abreast mass, tuberculosis is not often mentioned especially when there is 
no other tuberculosis focus. In both cases histology enables to correct diagnosis.

\section{Conclusion}

The most common site of extra-pulmonary tuberculosis explored by imaging is pleural followed ganglionic, osteo-articular and abdominal locations. It affects young adults with a male discrete predominance in our series. The co-infection TB-HIV is particularly high in our series, confirming that HIV has changed tuberculosis face especially extra-pulmonary tuberculosis. Medical scanning based on conventional radiography, ultrasonography and CT-scan is indispensable for the diagnosis and the care of extra-pulmonary tuberculosis since some locations may be confused with many others and lead to diagnosis errors and delay treatment.

\section{Conflict of Interest}

No.

\section{References}

[1] Awasthi, S., Saxena, M., Ahmad, F., Kumar, A. and Dutta, S. (2015) Abdominal Tuberculosis in a Tertiary Teaching Hospital: Review of 48 Cases. Journal of Clinical and Diagnostic Research, 9, EC01-EC03

[2] Park, K, Ed. (2007) Park’s Textbook of Preventive \& Social Medicine. 19 Edition, M/S Banarasi das Bhanot, Jabalpur, p. 768.

[3] Ravolamanana Ralisata, L., Rabenjamina, F.R. and Ralison, A. (2000) Extrathoracic Tuberculosis in Mahajanga Hospital (Madagascar). Archives de l'Institut Pasteur de Madagascar, 66, 13-17.

[4] Darré, T., Tchaou, M., Sonhaye, L., Patassi, A.A., Kanassoua, K., Tchangai, B., Amegbor, K. and Napo-Koura, G. (2015) Analysis of a Series of 44 Cases of Peritoneal Tuberculosis Diagnosed in the Pathology Laboratory of the Tokoin Teaching Hospital of Lomé (1993-2014). Bulletin de la Société de pathologie exotique, 108, 324-327. http://dx.doi.org/10.1007/s13149-015-0458-x

[5] World Health Organization (WHO) (2012) WHO Policy on Collaborative TB/HIV Activities Guidelines for National Programmes and Other Stakeholders. World Health Organization, Geneva, 36 p. http://www.who.int/tb/publications/2012/tb_hiv_policy_9789241503006/en/

[6] Ménard, D., Pécarrère, J.L., Ramaroson, F., Lesbordes, J.L., Andrianarisoa, R., Razafitsiarovana, I., Andriamiandrisoa, M.L., Raholimina Rahary, V., Rakotonizao, J., Richard, J., Peghini, M., Guyon, P. and Chanteau, S. (1995) ExtraPulmonary Tuberculosis in Antananarivo. Principal Localizations and Biological Diagnosis. Archives de l'Institut Pasteur de Madagascar, 62, 77-82.

[7] Yoon, H.J., Song, Y.G., Park, I.W., Choi, J.P., Chang, K.H. and Kim, J.M. (2004) Clinical Manifestations and Diagnosis of Extrapulmonary Tuberculosis. Yonsei Medical Journal, 45, 453-461. http://dx.doi.org/10.3349/ymj.2004.45.3.453

[8] Paustian, F.F. and Marshall, J.B. (1985) Intestinal Tuberculosis. In: Berk, J.E., ed., Bockus Gastroenterology, 4th Edition, WB Saunders, Philadelphia, 2018-2036.

[9] Davidson, P.T. and Horowitz, I. (1970) Skeletal Tuberculosis: A Review with Patient Presentations and Discussion. American Journal of Medicine, 48, 77-84. http://dx.doi.org/10.1016/0002-9343(70)90101-4

[10] Sankaran, B. (1993) Tuberculosis of Bones and Jonts-Oration. Indian Journal of Tuberculosis, 40, 109-118.

[11] Jutte, P.C., Van Loenhout-Rooyackers, J.H., Borgdorff, M.W. and van Horn, J.R. (2004) Increase of Bone and Joint Tuberculosis in the Netherlands. Journal of Bone Joint Surgery (British Volume), 86, 901-904. http://dx.doi.org/10.1302/0301-620X.86B6.14844

[12] Haider, A.L.M. (2007) Bones and Joints Tuberculosis. Bahrain Medical Bulletin, 29, 1-9.

[13] Sharma, S.K. and Mohan, A. (2004) Extrapulmonary Tuberculosis. Indian Journal of Medical Research, 120, $316-353$.

[14] Watts, H.G. and Lifeso, R.M. (1996) Current Concepts Review: Tuberculosis of Bones and Joints. The Journal of Bone and Joint Surgery, 78, 288-298.

[15] Muangchan, C. and Nilganuwong, S. (2009) The Study of Clinical Manifestation of Osteoarticular Tuberculosis in Siriraj Hospital, Thailand. Journal of the Medical Association of Thailand, 92, S101-S109.

[16] Blanie, M., Pellegrin, J.L. and Maugein, J. (2005) Contribution of PCR in the Diagnosis of Extrapulmonary Tuberculosis. Médecine et Maladies Infectieuses, 35, 17-22. http://dx.doi.org/10.1016/j.medmal.2004.08.002

[17] Malick, A. and Saxena, N.C. (2003) Ultrasound in Abdominal Tuberculosis. Abdominal Imaging, 28, 574-579. http://dx.doi.org/10.1007/s00261-002-0061-z 
[18] Pereira, J.M., Madureira, A.J., Vieira, A. and Ramos, I. (2005) Abdominal Tuberculosis: Imaging Features. European Journal of Radiology, 55, 173-180. http://dx.doi.org/10.1016/j.ejrad.2005.04.015

[19] Nassar, I., Errabih, I., Bouklata, S., Hammani, L., Krami, H.E., Lola, N., et al. (2008) Primary Tuberculosis of the Liver: Ten Cases Reports. Feuillets de Radiologie, 48, 203-207. http://dx.doi.org/10.1016/S0181-9801(08)74058-2

[20] N'goran, K., Akaffou, E., Konan, A.N. and N'goan-Domoua, A.M. (2011) Miliary Hepatic: An Unusual Ultrasound Presentation of Liver Tuberculosis Discover in an Immuno-Competent Adolescent. The Pan African Medical Journal, 9, 1.

[21] Mahi, M., Chaouir, S., Amil, T., Hanine, A. and Benameur, M. (2002) Isolated Tuberculosis of the Spleen. Report of a Case. J Radiol., 83, 479-481.

[22] Chong, V.H. and Lim, K.S. (2009) Gastrointestinal Tuberculosis. Singapore Medical Journal, 50, 638-645.

[23] Joual, A., Rabii, R., Guessous, H., Benjelloun, M., El Mrini, M. and Benjelloun, S. (2002) Isolated Testicular Tuberculosis: A Case Report. Annales d'urologie, 34, 192-194

[24] Agoda-Koussema, L.K., Tchaou, M., Adjénou, V., Sonhaye, L., Anoukoum, T., Tengué, K., Amouzou, K. and N’Dakena, K. (2011) Hetrogeneous Testicle on Ultrasonography: Consider Tuberculosis after Cancer in Endemic Zone. Médecine Tropicale, 71, 100. 